\title{
Associação entre sarcopenia e qualidade de vida relacionada à saúde em idosos comunitários
}

\section{Association between sarcopenia and health-related quality of life in community-dwelling older adults} Relación entre sarcopenia y calidad de vida respecto a la salud de adultos mayores de una comunidad

Camila Santos Chagas ${ }^{1}$ (D) https://orcid.org/0000-0002-5813-9799

Daniela Gonçalves Ohara ${ }^{1}$ id https://orcid.org/0000-0001-7055-6168

Areolino Pena Matos ${ }^{1}$ id https://orcid.org/0000-0002-3437-5105

Mônica Silvia Rodrigues de Oliveira ${ }^{1}$ iD https://orcid.org/0000-0002-2632-3639

Manoela Gomes Reis Lopes ${ }^{1}$ iD https://orcid.org/0000-0003-4262-2871

Flavia Aparecida Dias Marmo² ic https://orcid.org/0000-0001-6417-5748

Ana Carolina Pereira Nunes Pinto ${ }^{1}$ id https://orcid.org/0000-0002-1505-877X

Maycon Sousa Pegorari1 id hitps://orcid.org/0000-0003-4015-9895

Como citar:

Chagas CS, Ohara DG, Matos AP, Oliveira MS, Lopes MG, Marmo FA, et al. Associação entre sarcopenia e qualidade de vida relacionada à saúde em idosos comunitários. Acta Paul Enferm. 2021;34:AAPE002125.

DOI

http://dx.doi.org/10.37689/actaape/2021A0002125

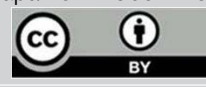

Idoso: Oualidade de

Keywords

Aged; Health of the elderly; Quality of life;

Sarcopenia

Descriptores

Anciano; Calidad de vida; Salud del anciano;

Sarcopenia

\section{Submetido}

4 de Agosto de 2020

Aceito

8 de Março de 2021

Autor correspondente

Maycon Sousa Pegorari

E-mail: mayconpegorari@yahoo.com.br

\section{Resumo}

Objetivo: 0 objetivo deste estudo foi comparar a qualidade de vida entre idosos sarcopênicos e não sarcopênicos e verificar a associação entre sarcopenia e qualidade de vida em idosos residentes na comunidade.

Métodos: Estudo transversal conduzido com idosos residentes na comunidade $(n=378)$ de Macapá, Amapá, Brasil. A qualidade de vida foi avaliada por meio do Short Form (36) Health Survey. 0 algoritmo proposto pelo Grupo de Trabalho Europeu sobre Sarcopenia em Pessoas Idosas (EWGSOP) foi usado para avaliar a sarcopenia. Foram realizadas análises descritivas, inferenciais e modelo de regressão linear.

Resultados: Os idosos sarcopênicos apresentaram escores de qualidade de vida significativamente mais baixos nos domínios função física, dor corporal, estado geral de saúde e função social. Após 0 ajuste, a sarcopenia associou-se inversamente ao funcionamento físico $(\beta=-0,125 ; p=0,010)$ e ao estado geral de saúde $(\beta=-0,112 ; p=0,028)$.

Conclusão: Os resultados deste estudo sugerem um provável declínio na qualidade de vida em idosos sarcopênicos, principalmente nos domínios funcionamento físico e estado geral de saúde.

\section{Abstract}

Objective: This study aimed to compare quality of life between sarcopenic and non-sarcopenic older adults and to verify the association of sarcopenia and quality of life in community-dwelling older adults.

Methods: This was a cross-sectional study conducted in community-dwelling older adults $(n=378)$ from Macapá, Amapá, Brazil. Quality of life was assessed using the Short Form (36) Health Survey. The algorithm proposed by the European Working Group on Sarcopenia in Older People (EWGSOP) was used to assess sarcopenia. Descriptive, inferential analyses and linear regression model were performed.

Results: Sarcopenic older adults presented significantly lower quality of life scores in the domains of physical functioning, bodily pain, general health status, and social functioning. After adjustment, sarcopenia was inversely associated with physical functioning $(\beta=-0.125 ; p=0.010)$ and general health status $(\beta=-0.112$; $p=0.028)$.

Conclusion: The results of this study suggest a probable decline in the quality of life in sarcopenic older adults, especially in the physical functioning and general health status domains.

\section{Resumen}

Objetivo: El objetivo de este estudio fue comparar la calidad de vida entre adultos mayores con sarcopenia y sin sarcopenia y verificar la relación entre sarcopenia y calidad de vida en adultos mayores residentes de la comunidad. 
Métodos: Estudio transversal llevado a cabo con adultos mayores residentes de la comunidad $(n=378)$ de Macapá, estado de Amapá, Brasil. La calidad de vida fue evaluada mediante el Short Form (36) Health Survey. El algoritmo propuesto por el Grupo Europeo de Trabajo sobre la Sarcopenia en Personas de Edad Avanzada (EWGSOP) fue utilizado para evaluar la sarcopenia. Se realizaron análisis descriptivos, inferenciales y modelo de regresión lineal.

Resultados: Los adultos mayores con sarcopenia presentaron una puntuación de calidad de vida significativamente más baja en los dominios función física, dolor corporal, estado general de salud y función social. Luego del ajuste, la sarcopenia se relacionó inversamente con el funcionamiento físico ( $\beta=-0,125$; $p=0,010)$ y con el estado general de salud $(\beta=-0,112 ; p=0,028)$.

Conclusión: Los resultados de este estudio sugieren un probable deterioro en la calidad de vida de adultos mayores con sarcopenia, principalmente en los dominios funcionamiento físico y estado general de salud.

\section{Introdução}

O envelhecimento é um processo caracterizado pelo acúmulo de danos moleculares e celulares ao longo da vida que, fundamentalmente, leva a uma diminuição progressiva das reservas fisiológicas, da massa muscular e da capacidade funcional. ${ }^{(1)}$ Em última análise, esses processos associados ao envelhecimento resultam em um risco maior de muitas doenças e morte. ${ }^{(1)}$ A perda de massa muscular é uma alteração especialmente grave associada ao envelhecimento. ${ }^{(2)}$ Em adultos jovens, a massa muscular magra compreende até cerca de $50 \%$ do peso corporal total, mas diminui para aproximadamente $25 \%$ ao entrar na faixa etária de 75-80 anos. ${ }^{(3)} \mathrm{A}$ perda muscular relacionada à idade é uma espiral descendente que pode levar à diminuição da força e da funcionalidade muscular. ${ }^{(2,4)}$ A perda simultânea de massa e força muscular que ocorre com o avanço da idade foi denominada sarcopenia. ${ }^{(2,4)}$

A sarcopenia é um fenômeno generalizado com uma etiologia complexa e multifatorial. ${ }^{(5)}$ Os fatores mais provavelmente envolvidos são estado nutricional, atividade física, alteraçóes hormonais, alteraçôes nas citocinas pró-inflamatórias circulantes e hereditariedade genética. ${ }^{(5)}$ Embora a sarcopenia tenha sido estudada há muito tempo ${ }^{(5)}$ e numerosas ferramentas para avaliar a massa muscular e função estejam disponíveis atualmente, ${ }^{(6)}$ os múltiplos mecanismos fisiopatológicos subjacentes à etiologia da sarcopenia não foram totalmente descritos. A detecçáo precoce da sarcopenia continua desafiadora, e várias definiçóes foram propostas para facilitar a detecção e o diagnóstico. ${ }^{(2,7,8)}$

A prevalência de sarcopenia depende de sua definição e varia entre 5 e $13 \%$ em pessoas de 60 a 70 anos e 11 a $50 \%$ em pessoas com mais de 80 anos. $^{(9)}$ Além de sua alta prevalência em idosos, vários efeitos da sarcopenia são indicadores prognósticos na carga de saúde pública. ${ }^{(2)}$ A sarcopenia está independentemente associada ao comprometimento funcional, deficiência física, quedas, fraturas, hospitalização, fragilidade, aumento dos gastos com saúde e prevê mortalidade por todas as causas entre idosos. ${ }^{(10)}$ A prevalência e o impacto da sarcopenia no sistema de saúde são especialmente relevantes em vista do aumento da expectativa de vida global. ${ }^{(2)}$

Embora o envelhecimento da população global seja um fenômeno positivo, ele é acompanhado por um aumento significativo na prevalência de condições adversas, como a sarcopenia. Portanto, impóe grandes desafios à saúde pública. ${ }^{(11)}$ Como a mortalidade é uma realidade iminente em idades mais avançadas, o foco dos cuidados de saúde não deve mais ser apenas prolongar a duração da vida, mas também melhorar a qualidade de vida. ${ }^{(12)}$ A qualidade de vida em pessoas idosas inclui desafios relacionados à independência, interação social, saúde e capacidade de continuar levando uma vida plena. ${ }^{(13)} \mathrm{A}$ Organização Mundial da Saúde enfatiza que manter a qualidade de vida durante o envelhecimento é um objetivo fundamental tanto para os indivíduos quanto para os legisladores. ${ }^{(11)}$

A relação entre sarcopenia e qualidade de vida já foi estudada anteriormente. ${ }^{(14,17)}$ Embora alguns estudos tenham constatado que a sarcopenia está negativamente associada à qualidade de vida, principalmente nos domínios da saúde física, ${ }^{(14,15)}$ outros estudos não identificaram a mesma associação. ${ }^{(16,17)}$ Além disso, uma revisão sistemática anterior ${ }^{(12)}$ identificou apenas um estudo sobre a associação entre sarcopenia e qualidade de vida que considerou uma definição do consenso de sarcopenia. ${ }^{(18)}$ Esta revisão sistemática destacou a necessidade de estudos de boa qualidade com grandes tamanhos de amostra usando uma definição de consenso de sarcopenia para esclarecer a 
associação entre os diferentes domínios da qualidade de vida e sarcopenia. ${ }^{(12)}$ A relação entre sarcopenia e qualidade de vida tem sido uma preocupação em diferentes contextos, como em instituiçóes de longa permanência, ${ }^{(19)}$ em ambulatórios, ${ }^{(20)}$ e na comunidade. ${ }^{(21)}$ A compreensão completa do impacto sobre a qualidade de vida decorrente da sarcopenia ajudará a fornecer aos pesquisadores da área da saúde as evidências necessárias para apoiar futuros estudos de intervenção. Além disso, para profissionais de saúde, como médicos, enfermeiras e fisioterapeutas, uma melhor compreensão das condiçóes que podem afetar a qualidade de vida dos idosos pode fornecer informações úteis para basear uma avaliação adequada desta população. Assim, este estudo teve como objetivo comparar a qualidade de vida de idosos sarcopênicos e não sarcopênicos e verificar a associação entre sarcopenia e qualidade de vida em idosos residentes na comunidade.

\section{Métodos}

\section{Desenho e população do estudo}

Este estudo transversal ocorreu na área urbana de Macapá em 2017, um município da região amazônica brasileira. $\mathrm{O}$ estudo foi aprovado (protocolo $\mathrm{n}^{\mathrm{o}}$ 1.738.671) pelo comitê local de ética em pesquisa em humanos. Outros resultados deste estudo foram relatados anteriormente. ${ }^{(22)}$

Para estimar o tamanho da amostra necessária, utilizou-se a informação disponibilizada pelo Instituto Brasileiro de Geografia e Estatística (IBGE) referente à população residente na zona urbana de Macapá em 2010. ${ }^{(23)}$ Portanto, considerando uma populaçáo de 19.955 das pessoas com 60 anos ou mais residentes na zona urbana de Macapá, ${ }^{(23)}$ assumindo $50 \%$ de prevalência de problemas de saúde nesta população, precisão de $5 \%$ e intervalo de confiança de 95\%, 377 indivíduos foram considerados necessários para compor uma amostra representativa neste estudo. Os participantes foram recrutados aleatoriamente em um processo de amostragem por conglomerados em dois estágios. Primeiramente, foram considerados os setores censitários com informaçôes também fornecidas pelo IBGE sobre os bairros e ruas, os quais foram sorteados para posterior identificação dos idosos nas residências. Os participantes foram cadastrados e avaliados em seus respectivos domicílios. Estudantes de graduação em fisioterapia devidamente treinados e acompanhados por supervisores de campo (pesquisadores e professores) realizaram as entrevistas presenciais. ${ }^{(24)}$

Foram incluídos indivíduos com 60 anos ou mais de idade que andavam [com ou sem dispositivos de auxílio à locomoção]. Foram excluídos os indivíduos não localizados após três tentativas, que se mudaram da cidade, hospitalizados, apresentaram sequelas neurológicas, não conseguiram responder às perguntas da entrevista ou realizar os testes ou apresentaram declínio cognitivo. O Mini-Exame do Estado Mental foi usado para examinar todos os possíveis participantes quanto ao declínio cognitivo. O mini Exame Mental foi traduzido e validado para uso no Brasil, ${ }^{(25)}$ que ajusta o ponto de corte à escolaridade do paciente.

\section{Qualidade de vida (variável dependente)}

A versão de 1992 do Short Form (36) Health Survey, traduzida e validada para uso no Brasil (26), foi utilizada para avaliar a qualidade de vida. O Short Form-36 é composto por 36 itens divididos em oito domínios: funcionamento físico, limitações de função devido à saúde física (abreviado como "função física"), limitações de função devido a problemas emocionais ("função emocional"), dor corporal, geral estado de saúde, vitalidade (também conhecido como "energia/fadiga"), funcionamento social e saúde mental (ou "bem-estar emocional"). Os escores variam de 0 a 100 em cada componente, com escores mais altos representando melhor estado de saúde. ${ }^{(26)}$

\section{Sarcopenia (variável independente)}

A definição proposta pelo Grupo de Trabalho Europeu sobre Sarcopenia em Idosos (EWGSOP) foi utilizada para avaliar a sarcopenia. ${ }^{(7)} \mathrm{A}$ massa muscular foi avaliada por meio da equação de massa muscular total proposta em estudo anterior ${ }^{(27)}$ validado para uso em idosos brasileiros. ${ }^{(28)} \mathrm{O}$ índice de massa muscular foi calculado dividindo a massa muscular total de uma pessoa por sua altura. Massa muscular baixa foi defi- 
nida como aquela dentro do percentil 20 inferior da amostra no índice de massa corporal, ${ }^{(29,30}$ ) que correspondeu a valores abaixo de $9,61 \mathrm{~kg} / \mathrm{m}^{2}$ para homens e $6,92 \mathrm{~kg} / \mathrm{m}^{2}$ para mulheres. A avaliação da força muscular e do desempenho físico foi realizada por meio de métodos recomendados pelo EWGSOP(7) Para a avaliação da força muscular, foi utilizado um dinamômetro manual hidráulico (modelo SH5001, SAEHAN ${ }^{\circ}$ ). A média de três medidas tomadas em intervalos de um minuto no teste de força de preensão manual foi usada para análise estatística. A baixa força muscular foi categorizada em valores abaixo de $30 \mathrm{~kg} /$ força [kg/f] para homens e abaixo de $20 \mathrm{~kg} / \mathrm{f}$ para mulheres, e o desempenho físico foi avaliado com base no tempo para percorrer uma distância de quatro metros em um teste de velocidade da marcha. ${ }^{(7)} \mathrm{O}$ desempenho físico foi considerado prejudicado se o participante concluísse o teste a uma velocidade de caminhada confortável de $0,8 \mathrm{~m} / \mathrm{s}$ ou mais lenta. ${ }^{(7)}$

Tabela 1. Características de idosos residentes na comunidade com e sem sarcopenia e amostra total $(n=378)$

\begin{tabular}{l|c|c|c|c}
\hline Variáveis & $\begin{array}{c}\text { Sarcopênico } \\
(\mathbf{n = 4 8 )}\end{array}$ & $\begin{array}{c}\text { Não } \\
\text { sarcopênico } \\
(\mathbf{n = 3 3 0 )}\end{array}$ & $\begin{array}{c}\text { Valor } \\
\text { de } \mathbf{p}^{*}\end{array}$ & $\begin{array}{c}\text { Amostra total } \\
(\mathbf{n = 3 7 8})\end{array}$ \\
\hline \begin{tabular}{l|c|c|c|} 
Idade (anos) \\
Gênero (n/\%)
\end{tabular} & $77,31 \pm 7,95$ & $68,98 \pm 6,59$ & $<0,001$ & $70,04 \pm 7,31$ \\
\hline Masculino & $14(29,16)$ & $116(35,15)$ & 0,415 & $130(34,39)$ \\
\hline Feminino & $34(70,84)$ & $214(64,85)$ & & $248(65,61)$ \\
\hline Escolaridade (anos) & $4,16 \pm 4,04$ & $6,08 \pm 5,34$ & 0,005 & $5,83 \pm 5,23$ \\
\hline Altura (m) & $1,51 \pm 0,86$ & $1,54 \pm 0,88$ & 0,006 & $1,54 \pm 0,89$ \\
\hline Peso (Kg) & $50,69 \pm 7,66$ & $68,99 \pm 12,37$ & $<0,001$ & $66,67 \pm 13,34$ \\
\hline IMC (kg/m²) & $22,19 \pm 2,29$ & $28,85 \pm 4,64$ & $<0,001$ & $28,01 \pm 4,94$ \\
\hline MMI (kg/m²) & $6,99 \pm 1,42$ & $9,18 \pm 1,61$ & $<0,001$ & $8,91 \pm 1,74$ \\
\hline HGS (Kgf) & $18,84 \pm 5,26$ & $25,52 \pm 9,19$ & $<0,001$ & $24,67 \pm 9,06$ \\
\hline Velocidade de caminhada & $0,79 \pm 0,30$ & $1,03 \pm 0,29$ & $<0,001$ & $0,99 \pm 0,31$ \\
\hline (0.8 m/s) & & & & \\
\hline Número de doenças & $5,50 \pm 2,32$ & $5,33 \pm 2,95$ & 0,713 & $5,35 \pm 2,87$ \\
\hline
\end{tabular}

Os dados são expressos como $\mathrm{n}=$ número de sujeitos; média + desvio padrão; $\mathrm{m}=$ metros; $\mathrm{Kg}=$ quilograma; IMC = índice de massa corporal; $\mathrm{MMI}=$ indice de massa muscular; $\mathrm{HGS}=$ força de preensão manual; $\mathrm{Kg} / \mathrm{f}=$ quilograma / força; * $\mathrm{p}<0,05$; Testes qui-quadrado et de Student.

\section{Análise dos dados}

A análise descritiva foi realizada por meio de frequências e porcentagens para variáveis categóricas; e médias e desvios-padrão para os contínuos. Para comparar participantes sarcopênicos e não sarcopênicos, um teste qui-quadrado foi usado para variáveis categóricas e um teste t de Student foi usado para variáveis contínuas. Para avaliar a associação entre os domínios da qualidade de vida e a sarcopenia, análises brutas e ajustadas foram realizadas por meio de um modelo de regressão linear. Os modelos foram ajustados para idade, sexo, escolaridade e número de doenças, sendo utilizados intervalos de confiança (IC) de $95 \%$ e nível de significância de 5\%. Foram considerados os pré-requisitos mínimos de normalidade, linearidade e homocedasticidade dos resíduos, bem como a ausência de multicolinearidade. Todos os dados foram analisados por meio da versão 21.0 do programa Statistical Package for the Social Sciences (SPSS).

\section{Resultados}

Trezentos e setenta e oito participantes constituíram a amostra final. Neste estudo, a maioria dos participantes $(65,61 \%)$ era do sexo feminino. A prevalência de sarcopenia nos participantes incluídos foi de $12,7 \%(\mathrm{n}=48)$. Os participantes sarcopênicos eram mais velhos e apresentavam média de anos de estudo mais baixa do que os idosos não sarcopênicos (Tabela 1).

Os idosos sarcopênicos apresentaram menores escores de qualidade de vida nos domínios funciomanento físico, dor corporal, estado geral de saúde e função social $(\mathrm{p}<0,05)$ (Tabela 2$)$.

Tabela 2. Domínios de qualidade de vida em idosos residentes na comunidade com e sem sarcopenia $(\mathrm{n}=378)$

\begin{tabular}{|c|c|c|c|c|c|}
\hline \multirow{3}{*}{$\begin{array}{l}\text { Variáveis } \\
\text { Short Form-36 (SF-36) }\end{array}$} & & & \multirow{3}{*}{ Valor de $\mathrm{p}^{\star}$} & \multirow{3}{*}{ Amostra total } & \multirow{3}{*}{ Cronbach's alpha } \\
\hline & Sim & Não & & & \\
\hline & \multicolumn{2}{|c|}{ média \pm sd } & & & \\
\hline Funcionamento físico & $68,02 \pm 26,15$ & $81,32 \pm 21,99$ & $<0,001$ & $79,63 \pm 22,96$ & 0,799 \\
\hline Função física & $65,63 \pm 41,45$ & $71,29 \pm 38,34$ & 0,345 & $70,57 \pm 38,74$ & 0,810 \\
\hline Dor corporal & $60,27 \pm 28,76$ & $69,01 \pm 27,16$ & 0,040 & $67,89 \pm 27,49$ & 0,806 \\
\hline Estado geral de saúde & $59,04 \pm 19,27$ & $64,12 \pm 14,93$ & 0,035 & $63,47 \pm 15,61$ & 0,810 \\
\hline Vitalidade & $71,35 \pm 23,19$ & $75,68 \pm 23,47$ & 0,233 & $75,13 \pm 23,45$ & 0,795 \\
\hline Função social & $79,68 \pm 27,36$ & $87,99 \pm 21,51$ & 0,017 & $86,94 \pm 22,47$ & 0,796 \\
\hline Função emocional & $73,61 \pm 40,06$ & $77,87 \pm 37,87$ & 0,469 & $77,34 \pm 38,01$ & 0,809 \\
\hline Saúde mental & $81,92 \pm 22,98$ & $84,36 \pm 19,16$ & 0,422 & $84,05 \pm 19,67$ & 0,798 \\
\hline
\end{tabular}


Tabela 3. Associação entre sarcopenia e qualidade de vida em idosos comunitários $(\mathrm{n}=378)$

\begin{tabular}{|c|c|c|c|c|c|c|c|c|}
\hline \multirow{3}{*}{$\begin{array}{l}\text { Variável } \\
\text { Short Form-36 }\end{array}$} & \multirow[b]{3}{*}{ B } & \multirow[b]{3}{*}{ Erro padrão } & \multirow[b]{3}{*}{$\beta$} & \multicolumn{2}{|c|}{ Sarcopenia } & & & \multirow[b]{3}{*}{$\mathrm{R}^{2}$} \\
\hline & & & & \multirow[b]{2}{*}{$\mathrm{T}$} & \multirow[b]{2}{*}{ Valor de $\mathrm{p}^{\star}$} & \multicolumn{2}{|c|}{$95 \% \mathrm{Cl}$} & \\
\hline & & & & & & $\begin{array}{l}\text { Limite } \\
\text { inferior }\end{array}$ & $\begin{array}{l}\text { Limite } \\
\text { Superior }\end{array}$ & \\
\hline \multicolumn{9}{|c|}{ Funcionamento físico } \\
\hline Não ajustado & $-13,297$ & 3,485 & $-0,193$ & $-3,816$ & $<0,001$ & $-20,149$ & $-6,445$ & 0,037 \\
\hline Ajustado & $-8,638$ & 3,353 & $-0,125$ & $-2,576$ & 0,010 & $-15,231$ & $-2,044$ & 0,248 \\
\hline \multicolumn{9}{|l|}{ Função física } \\
\hline Não ajustado & $-5,663$ & 5,985 & $-0,049$ & $-0,946$ & 0,345 & $-17,431$ & 6,105 & 0,002 \\
\hline Ajustado & $-2,988$ & 6,046 & $-0,026$ & $-0,494$ & 0,621 & $-14,877$ & 8,901 & 0,141 \\
\hline \multicolumn{9}{|l|}{ Dor corporal } \\
\hline Não ajustado & $-8,735$ & 4,229 & $-0,106$ & $-2,066$ & 0,040 & $-17,050$ & $-0,421$ & 0,011 \\
\hline Ajustado & $-7,878$ & 4,225 & $-0,096$ & $-1,865$ & 0,063 & $-16,186$ & 0,430 & 0,167 \\
\hline \multicolumn{9}{|c|}{ Estado geral de saúde } \\
\hline Não ajustado & $-5,083$ & 2,401 & $-0,109$ & $-2,117$ & 0,035 & $-9,804$ & $-0,361$ & 0,012 \\
\hline Ajustado & $-5,239$ & 2,380 & $-0,112$ & $-2,202$ & 0,028 & $-9,918$ & $-0,560$ & 0,181 \\
\hline \multicolumn{9}{|l|}{ Vitalidade } \\
\hline Não ajustado & $-4,328$ & 3,621 & $-0,062$ & $-1,195$ & 0,233 & $-11,447$ & 2,792 & 0,004 \\
\hline Ajustado & 0,145 & 3,578 & 0,002 & 0,041 & 0,968 & $-6,890$ & 7,180 & 0,179 \\
\hline \multicolumn{9}{|l|}{ Função social } \\
\hline Não ajustado & $-8,305$ & 3,449 & $-0,123$ & $-2,408$ & 0,017 & $-15,087$ & $-1,523$ & 0,015 \\
\hline Ajustado & $-6,161$ & 3,537 & $-0,091$ & $-1,742$ & 0,082 & $-13,116$ & 0,794 & 0,126 \\
\hline \multicolumn{9}{|c|}{ Função física/emocional } \\
\hline Não ajustado & $-4,268$ & 5,888 & $-0,037$ & $-0,725$ & 0,469 & $-15,845$ & 7,309 & 0,001 \\
\hline Ajustado & $-2,229$ & 6,187 & $-0,020$ & $-0,360$ & 0,719 & $-14,395$ & 9,936 & 0,069 \\
\hline \multicolumn{9}{|l|}{ Saúde mental } \\
\hline Não ajustado & $-2,447$ & 3,041 & $-0,041$ & $-0,805$ & 0,422 & $-8,427$ & 3,533 & 0,002 \\
\hline Ajustado & $-3,151$ & 3,034 & $-0,053$ & $-1,039$ & 0,300 & $-9,118$ & 2,815 & 0,161 \\
\hline
\end{tabular}

$B=$ coeficiente não padronizado; $\beta=$ coeficiente padronizado; $T$ = teste $t ; C$ = intervalo de confiança; $R^{2}=$ Coeficiente de determinaçãa; ${ }^{*} p<0,05$; Ajustado para idade, sexo, escolaridade e número de doenças.

Após o ajuste, a sarcopenia associou-se inversamente ao funcionamento físico $(\beta=-0,125 ; \mathrm{p}=$ $0,010)$ e ao estado geral de saúde $(\beta=-0,112 ; \mathrm{p}=$ 0,028) (Tabela 3).

\section{Discussão}

Este estudo identificou que idosos sarcopênicos apresentaram menores escores de qualidade de vida nos domínios função física, dor corporal, estado geral de saúde e função social. A sarcopenia foi inversamente associada com funcionamento físico, dor corporal, estado geral de saúde e funcionamento social. Após o ajuste para idade, sexo, escolaridade e número de doenças, a sarcopenia associou-se inversamente ao funcionamento físico e ao estado geral de saúde.

Em nossa amostra, $12,7 \%$ dos participantes tinham sarcopenia de acordo com a definição do EWGSOP. ${ }^{(7)}$ Esse achado está de acordo com uma revisão sistemática anterior que estimou uma prevalência de sarcopenia de $16 \%$ no Brasil, com base em critérios de baixa massa muscular e função. ${ }^{(31)}$ A prevalência de sarcopenia e suas características podem variar de acordo com o ambiente e o critério diagnóstico aplicado nas diferentes amostras de estudo. Até onde sabemos, apenas três estudos anteriores $^{(15,18,32)}$ usaram uma definição de consenso de sarcopenia para examinar a associação de sarcopenia e os componentes físicos e mentais da qualidade de vida em idosos. Esses estudos aplicaram a definição do $\mathrm{EWGSOP}^{(7)}$ a idosos residentes na comunidade.

Em nossa amostra, os escores de funcionamento físico, dor corporal, estado geral de saúde e função social foram piores em idosos sarcopênicos do que em idosos não sarcopênicos, e esses escores foram inversamente associados à sarcopenia. No entanto, após o ajuste, apenas o funcionamento físico e os escores do estado geral de saúde foram inversamente associados à qualidade de vida, sugerindo que esses componentes podem ser críticos na qualidade de vida dos idosos. Mais especificamente, uma quantidade importante dos piores escores de qualidade de vida $(24,8 \%$ na capacidade funcional e $18,1 \%$ no 
domínio saúde geral) foram relacionados à sarcopenia. Em consonância com nossos resultados, um estudo anterior identificou que, após o ajuste para características basais potenciais, pessoas sarcopênicas tinham pior funcionamento físico autorreferido do que seus pares não sarcopênicos. ${ }^{(15)}$ Outro estudo descobriu que participantes sarcopênicos também tinham pior funcionamento físico e escores de estado geral de saúde do que seus pares não sarcopêni$\cos ^{(18)}$, embora as análises não tenham sido ajustadas para variáveis de confusão. ${ }^{(18)}$

Em uma análise transversal, os autores do estudo perceberam que, após ajustes, a sarcopenia grave estava inversamente associada aos componentes físico e mental da qualidade de vida ${ }^{(32)}$, embora os resultados das associações de domínios específicos da qualidade de vida não fossem relatado. A associação de sarcopenia grave com o componente mental da qualidade de vida neste estudo ${ }^{(32)}$ sugere que esse componente pode piorar com a progressão da sarcopenia. No geral, esses achados sugerem que a sarcopenia está inversamente associada à saúde geral e ao funcionamento físico e pode afetar a saúde mental conforme a condição avança.

É importante ressaltar que dois estudos ${ }^{(16,17)}$ não identificaram associaçóes entre os componentes da sarcopenia e a qualidade de vida. No entanto, esses estudos tiveram tamanhos de amostra pequenos e não classificaram a sarcopenia com base em um consenso anterior. Juntos, esses resultados sugerem que o uso de uma definição de sarcopenia baseada em consenso operacional parece ser útil para classificar com precisão os idosos sarcopênicos com diferentes níveis de gravidade.

Curiosamente, em nosso estudo, os piores escores em idosos sarcopênicos foram aqueles relacionados ao domínio estado geral de saúde. $\mathrm{O}$ estado geral de saúde no Short Form-36 avalia a percepçáo dos participantes sobre seu próprio estado de saúde e suas crenças sobre a probabilidade de piorar. ${ }^{(33)}$ Esta avaliação se correlaciona com os componentes físicos e mentais da qualidade de vida, ${ }^{(33)}$ sugerindo que a sarcopenia tem um impacto global na qualidade de vida relacionada à saúde em idosos.

Em nossa amostra, os participantes sarcopênicos eram mais velhos e tinham menos anos de escolari- dade do que os participantes não sarcopênicos. Esses resultados não são surpreendentes, uma vez que há uma conhecida perda de massa e força muscular durante o processo de envelhecimento ${ }^{(2)}$ e idosos com baixa escolaridade apresentam maior risco de sarcopenia. ${ }^{(34)}$ No entanto, pouco se sabe sobre a relação entre escolaridade e sarcopenia. Parece razoável que indivíduos com maior nível de escolaridade sejam mais propensos a aderir a hábitos de vida saudáveis que podem prevenir a perda de massa e força muscular. No entanto, mais estudos são necessários para esclarecer o papel da escolaridade na sarcopenia.

Algumas limitaçóes do nosso estudo devem ser consideradas. Primeiro, seu desenho transversal limita a capacidade de estabelecer uma relação causal entre sarcopenia e reduçáo da qualidade de vida. Em segundo lugar, a maioria dos participantes incluídos neste estudo era do sexo feminino e havia diferenças entre os participantes com e sem sarcopenia. Os participantes sarcopênicos eram mais velhos e apresentavam menor média de anos de estudo do que os idosos náo sarcopênicos. Também não limitamos nossa amostra a indivíduos sem doenças. Para minimizar essas limitaçóes, foram realizadas análises ajustadas para idade, sexo, escolaridade e número de doenças. Terceiro, a equação usada para calcular a massa muscular, a equação de Lee, não é o padrão ouro para avaliar a sarcopenia. No entanto, como essa equação oferece um cálculo estimado sem exigir equipamentos caros, é amplamente utilizada e de fácil aplicação. Finalmente, nossa ferramenta de avaliação de qualidade de vida não é específica para idosos, enquanto alguns outros países agora têm questionários de qualidade de vida mais específicos para idosos. No entanto, o questionário que usamos é particularmente útil em virtude de sua simplicidade e é a medida de qualidade de vida relacionada à saúde em pacientes mais velhos, ${ }^{(35)}$ também permitindo comparaçóes entre pessoas em diferentes idades e a detecção de declínios na qualidade de vida em adultos mais velhos versus jovens.

Este estudo também tem vários pontos fortes. Primeiro, foi conduzido em uma amostra representativa de idosos bem caracterizados residentes na comunidade. Em segundo lugar, ratificou que o uso de uma definiçáo de consenso padronizada de sarcopenia é de particular importância e uso prático. Em terceiro lu- 
gar, destaca a possível relevância de detectar a sarcopenia precocemente em adultos mais velhos para prevenir a progressão da sarcopenia e maior deterioração na qualidade de vida. Novos estudos que avaliem o efeito do diagnóstico precoce da sarcopenia na qualidade de vida são necessários. Por fim, mostrou que domínios específicos da qualidade de vida devem ser considerados na avaliação, prevenção e tratamento da sarcopenia em idosos. Devido às consequências da sarcopenia no funcionamento físico, saúde geral e, em última instância, na saúde mental, estudos adicionais devem considerar a importância de estratégias de rastreamento para identificar precocemente a sarcopenia em idosos e devem se concentrar em estratégias preventivas para seu manejo. Embora haja fortes evidências de que as pessoas mais velhas estáo vivendo mais, determinar se os anos adicionais de idade são dominados por declínios rápidos na saúde física e mental e quais indivíduos correm o risco de experimentar limitaçóes de capacidade é crucial para o desenvolvimento de políticas. ${ }^{(1)}$

\section{Conclusão}

Os idosos sarcopênicos apresentaram menores escores de qualidade de vida nos domínios função física, dor corporal, estado geral de saúde e função social. Após o ajuste para idade, sexo, escolaridade e número de doenças, a sarcopenia foi inversamente associada ao funcionamento físico e ao estado geral de saúde. Domínios específicos da qualidade de vida, como capacidade funcional e estado geral de saúde, devem ser considerados na avaliação, prevenção e tratamento da sarcopenia em idosos.

\section{Agradecimentos}

Fundação de Amparo a Pesquisa do Estado do Amapá (FAPEAP, número 250.203.029 / 2016).

\section{Colaborações}

Todos os autores colaboraram com a concepçáo e desenho do estudo, interpretação dos dados, elabo- ração do artigo, revisão crítica do conteúdo intelectual e aprovação da versão final a ser publicada.

\section{Referências}

1. Rudnicka E, Napierała P, Podfigurna A, Męczekalski B, Smolarczyk R, Grymowicz M. The World Health Organization (WHO) approach to healthy ageing. Maturitas. 2020;139:6-11.

2. Cruz-Jentoft AJ, Bahat G, Bauer J, Boirie $Y$, Bruyère 0 , Cederholm T, Cooper C, Landi F, Rolland Y, Sayer AA, Schneider SM, Sieber CC, Topinkova E, Vandewoude M, Visser M, Zamboni M; Writing Group for the European Working Group on Sarcopenia in Older People 2 (EWGSOP2), and the Extended Group for EWGSOP2. Sarcopenia: revised European consensus on definition and diagnosis. Age Ageing. 2019;48(1):16-31. Erratum in: Age Ageing. 2019;48(4):601.

3. Short KR, Nair KS. The effect of age on protein metabolism. Curr Opin Clin Nutr Metab Care. 2000;3(1):39-44. Review.

4. Cruz-JentoftAJ,SayerAA.Sarcopenia.Lancet.2019;393(10191):263646. Erratum in: Lancet. 2019;393(10191):2590.

5. Fielding RA, Vellas B, Evans WJ, Bhasin S, Morley JE, Newman AB, et al. Sarcopenia: an undiagnosed condition in older adults. Current consensus definition: prevalence, etiology, and consequences. International working group on sarcopenia. J Am Med Dir Assoc. 2011;12(4):249-56.

6. Beaudart C, Rolland Y, Cruz-Jentoft AJ, Bauer JM, Sieber C, Cooper C, et al. Assessment of Muscle Function and Physical Performance in Daily Clinical Practice: a position paper endorsed by the European Society for Clinical and Economic Aspects of Osteoporosis, Osteoarthritis and Musculoskeletal Diseases (ESCE0). Calcif Tissue Int. 2019;105(1):114. Review.

7. Cruz-Jentoft AJ, Baeyens JP, Bauer JM, Boirie Y, Cederholm T, Landi F, Martin FC, Michel JP, Rolland Y, Schneider SM, Topinková E, Vandewoude M, Zamboni M; European Working Group on Sarcopenia in Older People. Sarcopenia: European consensus on definition and diagnosis: Report of the European Working Group on Sarcopenia in Older People. Age Ageing. 2010;39(4):412-23.

8. Muscaritoli M, Anker SD, Argilés J, Aversa Z, Bauer JM, Biolo G, et al. Consensus definition of sarcopenia, cachexia and pre-cachexia: joint document elaborated by Special Interest Groups (SIG) "cachexiaanorexia in chronic wasting diseases" and "nutrition in geriatrics". Clin Nutr. 2010;29(2):154-9.

9. Mayhew AJ, Amog K, Phillips S, Parise G, McNicholas PD, de Souza RJ, et al. The prevalence of sarcopenia in community-dwelling older adults, an exploration of differences between studies and within definitions: a systematic review and meta-analyses. Age Ageing. 2019;48(1):48-56.

10. Liu P, Hao Q, Hai S, Wang H, Cao L, Dong B. Sarcopenia as a predictor of all-cause mortality among community-dwelling older people: a systematic review and meta-analysis. Maturitas. 2017;103:16-22.

11. World Health Organization (WHO). Ageing. Geneva: WHO; 2021 [ cited 2021 Feb 20]. Available from: https://www.who.int/ageing/en/

12. Woo T, Yu S, Visvanathan R. Systematic Literature Review on the Relationship Between Biomarkers of Sarcopenia and Quality of Life in Older People. J Frailty Aging. 2016;5(2):88-99. Review.

13. Esteve-Clavero A, Ayora-Folch A, Maciá-Soler L, Molés-Julio MP. Factors associated with the quality of life of older people. Acta Paul Enferm. 2018;31:542-9. 
14. Silva Neto LS, Karnikowski MG, Osório NB, Pereira LC, Mendes MB, Galato D, et al. Association between sarcopenia and quality of life in quilombola elderly in Brazil. Int J Gen Med. 2016;9:89-97.

15. Beaudart C, Reginster JY, Petermans J, Gillain S, Quabron A, Locquet $M$, et al. Quality of life and physical components linked to sarcopenia: the SarcoPhAge study. Exp Gerontol. 2015;69:103-10.

16. Silva Neto LS, Karnikowiski MG, Tavares AB, Lima RM. Association between sarcopenia, sarcopenic obesity, muscle strength and quality of life variables in elderly women. Rev Bras Fisioter. 2012;16(5):360-7.

17. Sartor-Glittenberg C, Lehmann S, Okada M, Rosen D, Brewer K, Bay $\mathrm{RC}$. Variables explaining health-related quality of life in communitydwelling older adults. J Geriatr Phys Ther. 2014;37(2):83-91.

18. Patel HP, Syddall HE, Jameson K, Robinson S, Denison H, Roberts HC, et al. Prevalence of sarcopenia in community-dwelling older people in the UK using the European Working Group on Sarcopenia in Older People (EWGSOP) definition: findings from the Hertfordshire Cohort Study (HCS). Age Ageing. 2013;42(3):378-84.

19. Chang SF, Chiu SC. Effect of resistance training on quality of life in older people with sarcopenic obesity living in long-term care institutions: a quasi-experimental study. J Clin Nurs. 2020;29(13-14):2544-56.

20. Tan LF, Lim ZY, Choe R, Seetharaman S, Merchant R. Screening for Frailty and Sarcopenia Among Older Persons in Medical Outpatient Clinics and its Associations With Healthcare Burden. J Am Med Dir Assoc. 2017;18(7):583-7.

21. Verlaan S, Aspray TJ, Bauer JM, Cederholm T, Hemsworth J, Hill $\mathrm{TR}$, et al. Nutritional status, body composition, and quality of life in community-dwelling sarcopenic and non-sarcopenic older adults: a case-control study. Clin Nutr. 2017;36(1):267-74.

22. Ohara DG, Pegorari MS, Oliveira Dos Santos NL, de Fátima Ribeiro Silva C, Monteiro RL, Matos AP, et al. Respiratory Muscle Strength as a Discriminator of Sarcopenia in Community-Dwelling Elderly: A CrossSectional Study. J Nutr Health Aging. 2018;22(8):952-8.

23. Instituto Brasileiro de Geografia e Estatística (BBGE). Síntese de Indicadores Sociais: uma análise das condições de vida da população brasileira 2010. [Série: Estudos \& Pesquisa. Informação Demográfica e Socioeconômica, 27]. Rio de Janeiro: IBGE; 2010 [citado 2021 Fev 21]. Disponível em: https://biblioteca.ibge.gov.br/visualizacao/livros/liv45700.pdf

24. Silva CF, Pegorari MS, Matos AP, Ohara DG. Dyspnea is associated with poor physical performance among community-dwelling older adults: a populationbased cross-sectional study. Sao Paulo Med J. 2020;138:112-7.
25. Bertolucci PH, Brucki SM, Campacci SR, Juliano Y. O Mini-Exame do Estado Mental em uma população geral: impacto da escolaridade. Arq Neuro-Psiquiatr. 1994;52(1):1-7.

26. Ciconelli RM, Ferraz MB, Santos W, Meinao I, Quaresma MR. Tradução para a língua portuguesa e validação do questionário genérico de avaliação de qualidade de vida SF-36 (Brasil SF-36). Rev Bras Reumatol. 1999;39(3):143-50.

27. Lee RC, Wang Z, Heo M, Ross R, Janssen I, Heymsfield SB. Totalbody skeletal muscle mass: development and cross-validation of anthropometric prediction models. Am J Clin Nutr. 2000;72(3):796803. Erratum in: Am J Clin Nutr. 2001;73(5):995.

28. Rech CR, Dellagrana RA, Marucci MD, Petroski EL. Validity of anthropometric equations for the estimation of muscle mass in the elderly. Rev Bras Cineantropom Desempenho Hum. 2012;14(1)23-31.

29. Delmonico MJ, Harris TB, Lee JS, Visser M, Nevitt M, Kritchevsky SB, Tylavsky FA, Newman AB; Health, Aging and Body Composition Study. Alternative definitions of sarcopenia, lower extremity performance, and functional impairment with aging in older men and women. J Am Geriatr Soc. 2007;55(5):769-74.

30. Newman AB, Kupelian V, Visser M, Simonsick E, Goodpaster B, Nevitt M, Kritchevsky SB, Tylavsky FA, Rubin SM, Harris TB; Health ABC Study Investigators. Sarcopenia: alternative definitions and associations with lower extremity function. J Am Geriatr Soc. 2003;51(11):1602-9.

31. Diz JB, Leopoldino AA, Moreira BS, Henschke N, Dias RC, Pereira LS, et al. Prevalence of sarcopenia in older Brazilians: A systematic review and meta-analysis. Geriatr Gerontol Int. 2017;17(1):5-16.

32. Manrique-Espinoza B, Salinas-Rodríguez A, Rosas-Carrasco 0, Gutiérrez-Robledo LM, Avila-Funes JA. Sarcopenia Is Associated With Physical and Mental Components of Health-Related Quality of Life in Older Adults. J Am Med Dir Assoc. 2017;18(7):636.e1-636.e5.

33. Ware JE Jr, Gandek B. Overview of the SF-36 Health Survey and the International Quality of Life Assessment (IQOLA) Project. J Clin Epidemiol. 1998;51(11):903-12.

34. Brennan-Olsen S, Vogrin S, Balogun S, Wu F, Scott D, Jones G, et al. Education, occupation and operational measures of sarcopenia: Six years of Australian data. Australasian J Ageing. 2020;39;4(498505.

35. Hickey A, Barker M, McGee H, O'Boyle C. Measuring health-related quality of life in older patient populations: a review of current approaches. Pharmacoeconomics. 2005;23(10):971-93. Review. 